\title{
Fixed or adjustable sling in the treatment of male stress urinary incontinence: results from a large cohort study
}

\author{
Tanja Hüsch $^{1,2} \wedge$, Alexander Kretschmer ${ }^{3,4}$, Alice Obaje ${ }^{5}$, Ruth Kirschner-Hermanns ${ }^{6}$, Ralf Anding ${ }^{6}$, \\ Tobias Pottek ${ }^{7}$, Achim Rose ${ }^{8}$, Roberto Olianas ${ }^{9}$, Alexander Friedl ${ }^{10}$, Roland Homberg ${ }^{11}$, \\ Jesco Pfitzenmaier ${ }^{12}$, Rudi Abdunnur ${ }^{13}$, Fabian Queissert ${ }^{14}$, Carsten M. Naumann ${ }^{15}$, Josef Schweiger ${ }^{16}$, \\ Carola Wotzka ${ }^{17}$, Joanne Nyarangi-Dix ${ }^{18}$, Torben Hofmann ${ }^{19}$, Kurt Ulm ${ }^{20}$, Wilhelm Hübner ${ }^{21}$, \\ Ricarda M. Bauer ${ }^{3}$, Axel Haferkamp ${ }^{1}$; Debates On Male Incontinence (DOMINO)-Project
}

${ }^{1}$ Department of Urology and Pediatric Urology, University Medical Center of Johannes Gutenberg University, Mainz, Germany; ${ }^{2}$ Promedon GmbH, Clinical Research, Kolbermoor, Germany; ${ }^{3}$ Department of Urology, Ludwig-Maximilians-University, Munich, Germany; ${ }^{4}$ Vancouver Prostate Center, University of British Columbia, Vancouver, Canada; ${ }^{5}$ Department of Urology, St. Bernward Hospital Hildesheim, Hildesheim, Germany; ${ }^{6}$ Neuro-Urology/Urology and Pediatric Urology, University Hospital Bonn, Bonn, Germany; ${ }^{7}$ Reconstructive Urology, Vivantes Hospital, Berlin, Germany; ${ }^{8}$ Department of Urology and Pediatric Urology, Helios Hospital Duisburg, Duisburg, Germany; ${ }^{9}$ Department of Urology, Hospital Luneburg, Luneburg, Germany; ${ }^{10}$ Department of Urology, Merciful Sisters Hospital, Vienna, Austria; ${ }^{11}$ Department of Urology and Pediatric Urology, St. Barbara Hospital Hamm GmbH, Hamm, Germany; ${ }^{12}$ Department of Urology, Evangelic Hospital Bielefeld, Bielefeld, Germany; ${ }^{13}$ Department of Urology and Pediatric Urology, Helios Hospital Schwelm, Schwelm, Germany; ${ }^{14}$ Department of Urology, University Hospital Muenster, Muenster, Deutschland; ${ }^{15}$ Department of Urology and Pediatric Urology, Marienhaus Hospital Neuwied, Neuwied, Germany; ${ }^{16}$ Department of Urology and Pediatric Urology, Catholic Hospital St. Johann Nepomuk, Erfurt, Germany; ${ }^{17}$ Department of Urology, Diakonie Hospital Stuttgart, Stuttgart, Germany; ${ }^{18}$ Department of Urology and Pediatric Urology, University Hospital Heidelberg, Heidelberg, Germany; ${ }^{19}$ Department of Urology, Diakonie Hospital Schwaebisch Hall, Schwaebisch Hall, Germany; ${ }^{20}$ Institute of medical Statistic and Epidemiology, Technical University Munich, Munich, Germany; ${ }^{21}$ Department of Urology, Hospital Weinviertel Korneuburg, Korneuburg, Austria Contributions: (I) Conception and design: T Hüsch, A Kretschmer, A Obaje, R Anding, R Kirschner-Hermanns, T Pottek, A Rose, R Olianas, A Friedl, W Hübner, R Homberg, J Pfitzenmaier, R Abdunnur, F Queissert, CM Naumann, J Schweiger, C Wotzka, J Nyarangi-Dix, T Hofmann, K Ulm, RM Bauer, A Haferkamp; (II) Administrative Support: A Kretschmer; (III) Provision of study materials or patients: A Obaje, R Anding, R Kirschner-Hermanns, T Pottek, A Rose, R Olianas, A Friedl, W Hübner, R Homber, J Pfitzenmaier, R Abdunnur, F Queissert, CM Naumann, J Schweiger, C Wotzka, J Nyarangi-Dix, T Hofmann, RM Bauer, A Haferkamp; (IV) Collection and assembly of data: T Hüsch, A Kretschmer; (V) Data analysis and interpretation: T Hüsch, K Ulm; (VI) Manuscript writing: All authors; (VII) Final approval of manuscript: All authors.

Correspondence to: Tanja Hüsch, MD. Department of Urology and Pediatric Urology, University Medical Center of Johannes Gutenberg University Mainz, Langenbeckstr. 1, 55131 Mainz, Germany. Email: tanjahuesch@gmail.com.

\begin{abstract}
Background: Fixed and adjustable male slings for the treatment of male urinary stress incontinence became increasingly popular during the last decade. Although fixed slings are recommended for the treatment of mild to moderate stress urinary incontinence, there is still a lack of evidence regarding the precise indication for an adjustable male sling. Furthermore, there is still no evidence that one type of male sling is superior to another. However, both, adjustable and fixed slings, are commonly utilized in daily clinical practice. This current investigation aims to evaluate the differences between fixed and adjustable male slings regarding indications, complication rates and functional outcome in the treatment of male stress urinary incontinence in current clinical practice.

Methods: A total of 294 patients with a fixed and 176 patients with an adjustable male sling were evaluated in a multicenter single arm cohort trial. Data collection was performed retrospectively according the medical record. Functional outcome was prospectively analyzed by standardized, validated questionnaires. Descriptive statistics was performed to present patient characteristics, complication rates and functional outcome. A chi2-test for categorical and independent t-test for continuous variables was performed to
\end{abstract}

^, ORCID: 0000-0002-7872-5496. 
identify heterogeneity between the groups and to correlate preoperative characteristics with the outcome. A $\mathrm{P}$ value $<0.05$ was considered statistically significant.

Results: Patients with higher degree of urinary incontinence $(\mathrm{P}<0.001)$ and risk factors such as history of pelvic irradiation $(\mathrm{P}<0.001)$ or prior surgery for urethral stricture $(\mathrm{P}=0.032)$ were more likely to receive an adjustable MS. Complication rates were comparable except for infection $(\mathrm{P}=0.009,0$ vs. 2.3\%) and pain $(\mathrm{P}=0.001,1.7 \%$ vs. $11.3 \%)$ which occurred more frequently in adjustable slings. Functional outcome according validated questionnaires demonstrated no differences between fixed and adjustable male slings.

Conclusions: Adjustable male slings are more frequently utilized in patients with higher degree of incontinence and risk factors compared to fixed slings. No differences could be identified between functional outcome which may imply an advantage for adjustability. However, pain and infection rates were significantly higher in adjustable MS and should be considered in the decision process for sling type.

Keywords: Stress urinary incontinence; male; adjustable male sling; fixed male sling; quality of life

Submitted Dec 10, 2019. Accepted for publication May 14, 2020.

doi: $10.21037 /$ tau-19-852

View this article at: http://dx.doi.org/10.21037/tau-19-852

\section{Introduction}

Over the last decade, male slings (MS) for the treatment of male stress urinary incontinence (SUI) became increasingly popular (1). In general, sling surgery is a procedure with limited peri- and postoperative complications $(2,3)$. The most frequent complications are postoperative urinary retention and perineal pain (2) and the overall cure and improvement rates are described between $9 \%$ to $91.6 \%$ $(3,4)$. A sling procedure is recommended for mild to moderate urinary incontinence (5) although successful application has also been demonstrated in severe urinary incontinence (6).

MS are available as fixed and adjustable devices. As fixed slings seem to have demonstrated a gradual loss of efficacy over time, the idea of adjustability is the supplement of a compressive component to the relocating effect of the sling. Therefore, the regulation of the urethral closure pressure can be better adjusted to the individual patients' needs. Although clinical results from some cohort trials exist, randomized controlled trials are pending (3). So far, only one randomized study with a limited patient population exist (7) and furthermore, one prospective trial leaving the choice for an adjustable sling to the patient is available (8). Thus, the choice for a specific MS type depends predominantly on the surgeons' preference and experience. No evidence exists that adjustability offers an additional benefit over other types of slings or one sling is superior to another (5).

In order to compare the current indications and outcomes of fixed and adjustable MS in clinical daily practice, we investigated the complication rates and effectiveness of fixed and adjustable male slings in a multicentre cohort study. We present the following article in accordance with the STROBE reporting checklist (available at http://dx.doi. org/10.21037/tau-19-852).

\section{Methods}

The study was approved by the local ethics committee of Frankfurt university hospital (ethic vote number 442/13). We conducted a multicenter single arm cohort study including a total of 470 patients who have received a fixed or adjustable MS for male stress urinary incontinence in the study centers between 2010 and 2012. Patients were enrolled retrospectively in Germany and Austria, thus, selection criteria for a fixed or adjustable sling was not standardized. Baseline characteristics, perioperative data and complication rates were collected retrospectively according the medical record. Standardised and validated questionnaires were prospectively completed by the patients at time of enrolment. A signed informed consent was mandatory for this investigation. The following questionnaires were used: "Incontinence - Quality of Life" (9) (I-QoL; scale: 0-100, a higher score represents better QoL), "Patient Global Impression - Improvement" (10) (PGI-I; range $1-7$, very much better to very much worse), "International Consultation on Incontinence Questionnaire - Short Form" $(11,12)$ (ICIQ-SF; range: 0-21, 0: no incontinence, 1-5: slight, 6-12: moderate, 13-18: severe, 19-21: very severe), 
Table 1 Patients' baseline characteristics compared between fixed and adjustable slings

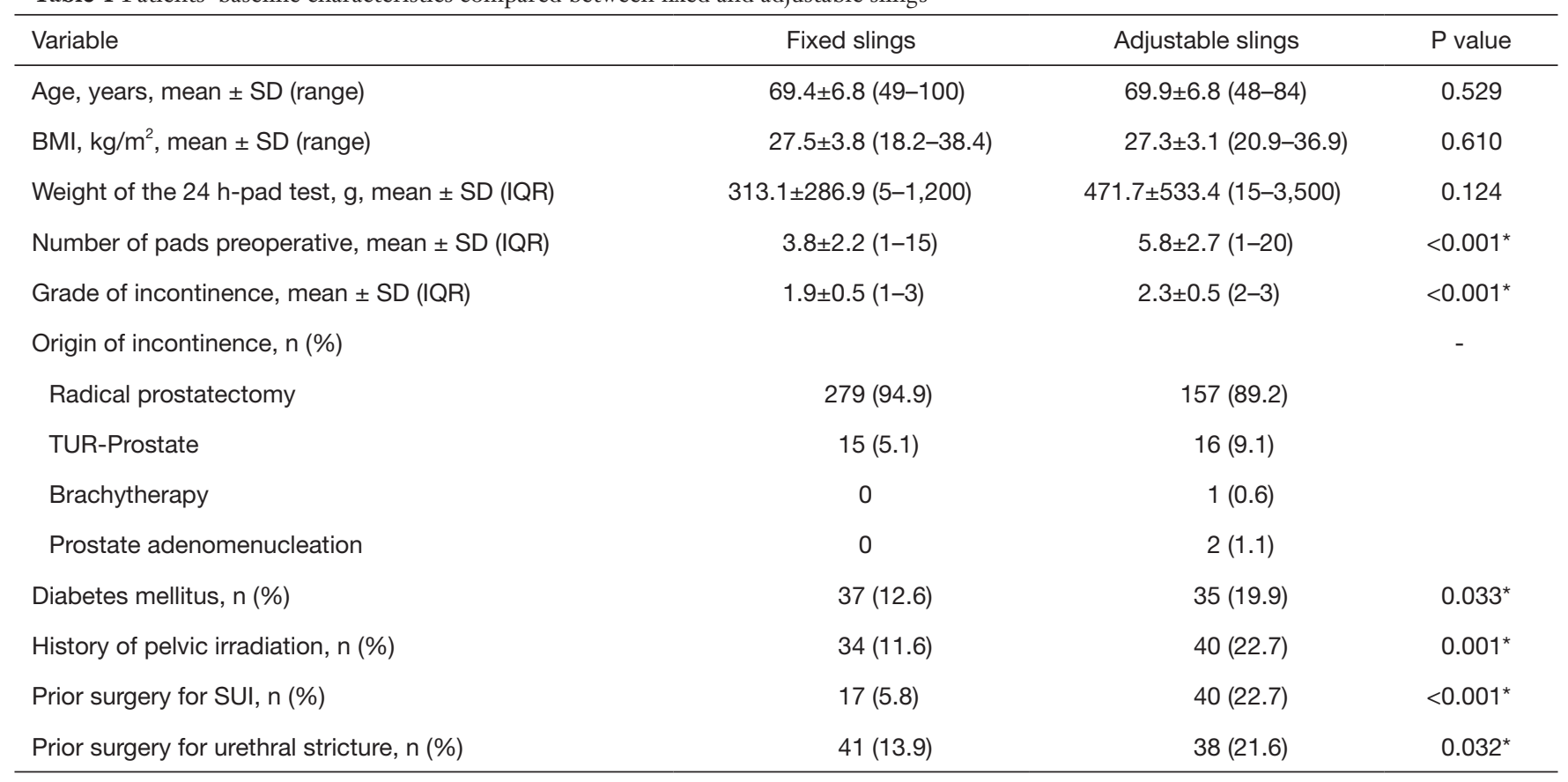

*, significance $\mathrm{P}<0.05$.

"Verbal Rating Scale of Pain" (VRS; range 0-10, a higher score represents more pain). Furthermore, the number of pads used per day and a 24-hour pad test were evaluated. Complications were evaluated according the Clavien Dindo Classification (13). Results were compared between the fixed and adjustable MS group. Functional outcome of fixed and adjustable MS group was additionally correlated with the confounders grade of incontinence (1-3), history of pelvic irradiation and prior surgery for urethral stricture disease. Additional subgroup analysis was performed in case of significant differences between the groups to identify confounder by utilized device. Quantitative variables were not grouped. Missing data have been handled in an available cases analysis approach.

\section{Statistical analysis}

Statistical analysis was performed by IBM SPSS (Armonk, New York, United States) Statistics Version 22 for Macintosh. Descriptive statistic was applied for presentation of population characteristics, complication rates and outcome. A chi ${ }^{2}$-test for categorical and independent t-test for continuous variables was performed to identify heterogeneity between the groups. Correlations of baseline categorial variables with continence outcome were performed by the chi ${ }^{2}$-test. A significance level of $5 \%$ was determined.

\section{Results}

A total of 294 (62.6\%) patients received a fixed and 176 (37.4\%) an adjustable MS. In the fixed MS group, 109 (37.1\%) and 185 (62.9\%) patients had an AdVance or AdvanceXP (Boston Scientific, Marlborough, Massachusetts, US) respectively. In the adjustable MS group, 127 (72.2\%) patients presented with an Argus classic or Argus-T (Promedon, Cordoba, Argentina) and 49 (27.8\%) with an ATOMS (AMI, Feldkirch, Austria). The baseline characteristics are presented in Table 1. Prior surgeries for SUI included other MS, bulking agents, ProAct (Uromedica, Plymouth, US) or artificial urinary sphincters.

The mean operation time ( $\mathrm{P}=0.036 ; 75.9$ vs. 70.3 minutes) was significantly longer for adjustable slings. Furthermore, significantly more intraoperative complications occurred in adjustable slings $(\mathrm{P}<0.001,0.3 \%$ vs. $10.2 \%)$. In subgroup analysis of adjustable MS, intraoperative complications occurred only in Argus classic $(\mathrm{P}<0.001)$ which included 17 perforations of the urinary bladder and one prolonged operation time due to scarred tissue. Regarding fixed sling, only one prolonged operation time because of scarred tissue 
Table 2 Postoperative complications in comparison between fixed and adjustable slings

\begin{tabular}{lcc}
\hline Variable & Fixed slings & Adjustable slings \\
\hline Clavien Dindo grade I, n (\%) & 0 & $2(1.1)$ \\
Bleeding & $23(7.8)$ & $13(7.4)$ \\
Urinary retention & & 0.067 \\
Clavien Dindo grade II, n (\%) & $2(0.7)$ & $5(2.8)$ \\
Impaired wound healing & $22(7.5)$ & $6(3.4)$ \\
DeNovo Urge & & 0.061 \\
Clavien Dindo grade III, n (\%) & 0 & $1(0.6)$ \\
Urethral erosion & 0 & $4(2.3)$ \\
Infection & & 0.196 \\
Clavien Dindo grade I and III, n (\%) & $5(1.7)$ & $21(11.9)$ \\
Pain & & $0.009^{*}$ \\
\hline
\end{tabular}

*, significance $\mathrm{P}<0.05$.

was reported. Postoperative complication rates according Clavien Dindo classification (13) are demonstrated in Table 2.

Infection occurred only in adjustable slings $(\mathrm{P}=0.009)$. In subgroup analysis of adjustable MS, no differences between Argus or ATOMS regarding infection rates could be identified $(\mathrm{P}=0.579)$. In three patients, Argus had to be removed completely and one patient with the ATOMS presented with infection of the port which had to be temporarily removed. Moreover, significantly more patients with an adjustable sling reported postoperative pain $(\mathrm{P}<0.001)$. In subgroup analysis of adjustable slings, more patients with Argus- $\mathrm{T}$ reported pain in comparison to Argus classic or ATOMS ( $\mathrm{P}<0.001,43.8 \%$ vs. $5.3 \%$ vs. $4.1 \%$ respectively).

Explantation occurred in $5(1.7 \%)$ and 7 (4.0\%) patients with fixed or adjustable MS group respectively $(\mathrm{P}=0.130)$. Argus- $T$ had to be removed in two patients due to persistent SUI and pain and in one patient due to washer-dislocation which impeded further adjustment of the sling. Argus classic was removed in one patient due to urethral erosion and in two patients due to infection. another. Unilateral transection of the sling had to be performed in four patients with a fixed sling due to hypercontinence with recurrent acute urinary retentions. One fixed sling had to be removed due to dislocation of the sling.

\section{Follow-up}

Follow-up was available in $126(42.9 \%)$ patients with fixed and $78(44.3 \%)$ with adjustable MS. The mean follow-up time was $41.4 \pm 13.5$ months for fixed and $36.9 \pm 13.3$ months for adjustable slings $(\mathrm{P}=0.421)$. Change of the continence device or additional secondary fixed MS due to persistent or recurrent incontinence occurred in 17 (5.8\%) patients with fixed and $16(9.1 \%)$ patients with adjustable MS. In fixed slings, nine patients received an additional fixed sling (Advance, AdvanceXP), three patients an adjustable MS (Remeex, Argus, Phorbas), five patients an artificial urinary sphincter and one patient underwent cystectomy with incontinent diversion because of recurrent urethral strictures. In adjustable slings, 11 patients received an artificial urinary sphincter and five patients a distinct adjustable MS (ATOMS, Argus).

Regarding functional outcome, no differences in ICIQSF, I-QoL (Figure 1) or PGI-I could be identified between adjustable and fixed slings (Table 3). There was no significant difference concerning the recommendation of the operation to a friend $(\mathrm{P}=0.523)$ or the willingness to repeat the surgery $(\mathrm{P}=0.797)$.

The mean pain rates were significantly higher in adjustable slings according the VRS of pain (Table 3). The mean difference in pain according VRS were $0.8,0.5$, 0.3 and 0.5 points regarding perineal (Figure 2), genital, symphyseal and inguinal pain respectively. In subgroup analysis, no significant differences could be identified between the devices.

Furthermore, no correlation between PGI-I and grade of incontinence $(\mathrm{P}=0.460)$, prior urethral stricture $(\mathrm{P}=0.450)$ or 


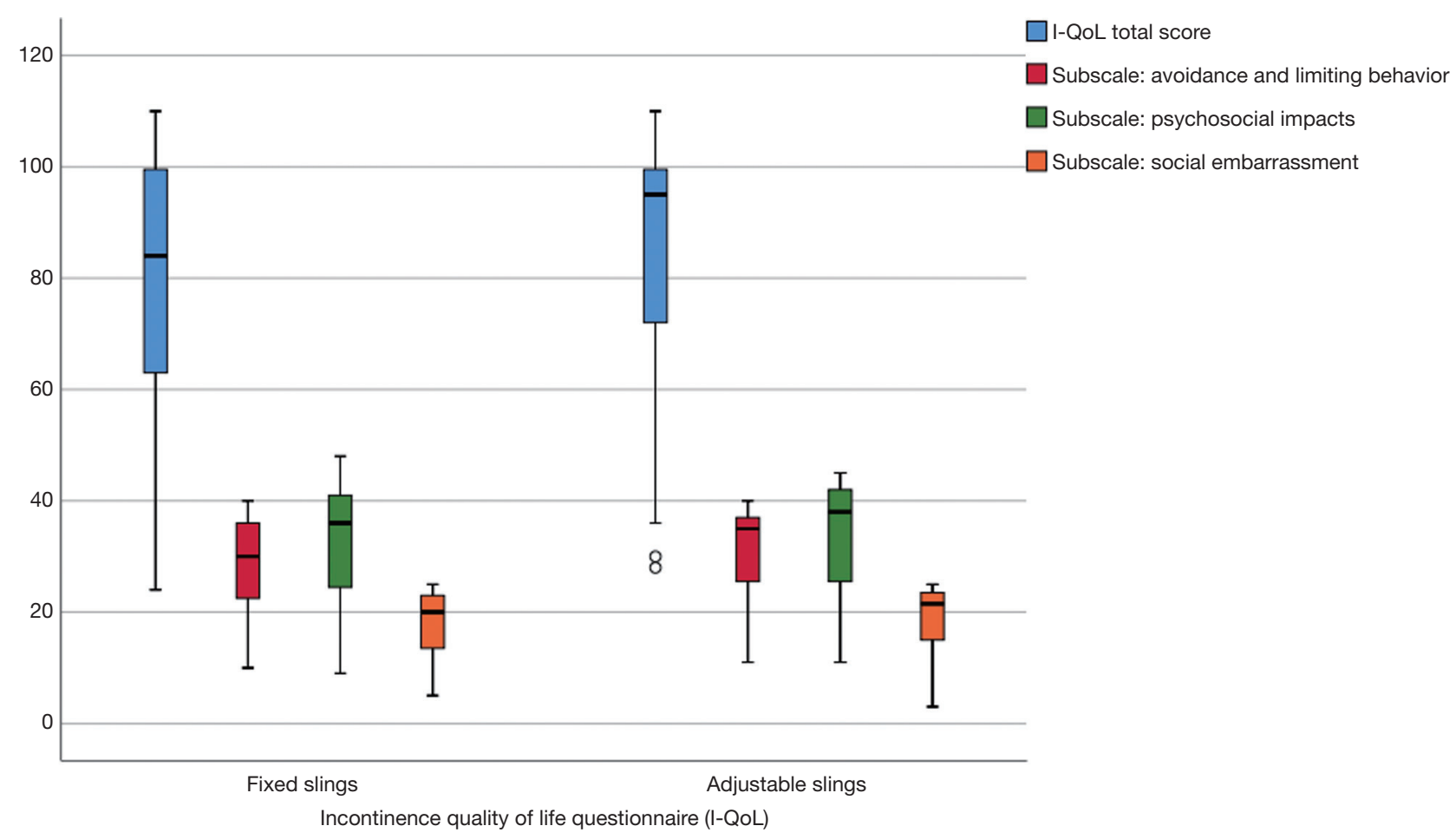

Figure 1 Comparison of the Incontinence Quality of Life Score and Subscales between fixed and adjustable slings.

a history of pelvic irradiation $(\mathrm{P}=0.427)$ could be identified in the adjustable MS group. In the fixed MS group, worse PGI correlated significantly with a history of urethral stricture disease $(\mathrm{P}=0.002)$. There was no correlation between a history of pelvic irradiation $(\mathrm{P}=0.589)$ or grad of incontinence at baseline $(\mathrm{P}=0.509)$.

\section{Discussion}

The present study investigated the differences in selection criteria, complication rates and outcome in comparison between fixed and adjustable MS for the treatment of male SUI in clinical daily practice. Although the indications for a fixed or an adjustable MS may be different in clinical practice, to our knowledge, only one randomised study with a limited patient population exist (7). There is still no evidence that one sling is superior to another and the additional benefit of adjustability remains unclear (5).

In preoperative selection criteria, we could demonstrate that patients with risk factors such as diabetes mellitus, history of pelvic irradiation, and prior urethral stricture disease are more likely to receive an adjustable male sling rather than a fixed sling. Furthermore, adjustable slings were more frequently offered secondary to prior failed surgical treatment of male SUI and even offered to patients with failed artificial urinary sphincter. This raises the question if whether or not this may imply a negative impact on the outcome of adjustable slings. Recent studies demonstrated low complication rates for male slings in general including adjustable male slings and the only independent risk factor for explantation was a history of pelvic irradiation (2). This is consistent with recent studies of fixed (6) or adjustable slings (14), reporting successful utilization even in patients with risk factors or high degree of incontinence. These results are consistent with the current trial which demonstrated comparable complications rates and functional outcome.

A problem with comparability between fixed and adjustable slings is the definition of the degree of urinary incontinence. Although, most studies suggest adjustable slings for moderate to severe and fixed slings for mild to moderate incontinence, the definitions vary widely. Depending on the study, the definition is either clinical according to the Stamey-classification, pad use per day (14) or by urine loss in the $24 \mathrm{~h}$-pad test (4). This implies a wide variety of incontinence degrees with different outcomes depending on the used definition. In the present study, the clinical definition by Stamey and the $24 \mathrm{~h}$ pad test were 
Table 3 Results of the questionnaires in comparison to fixed and adjustable slings

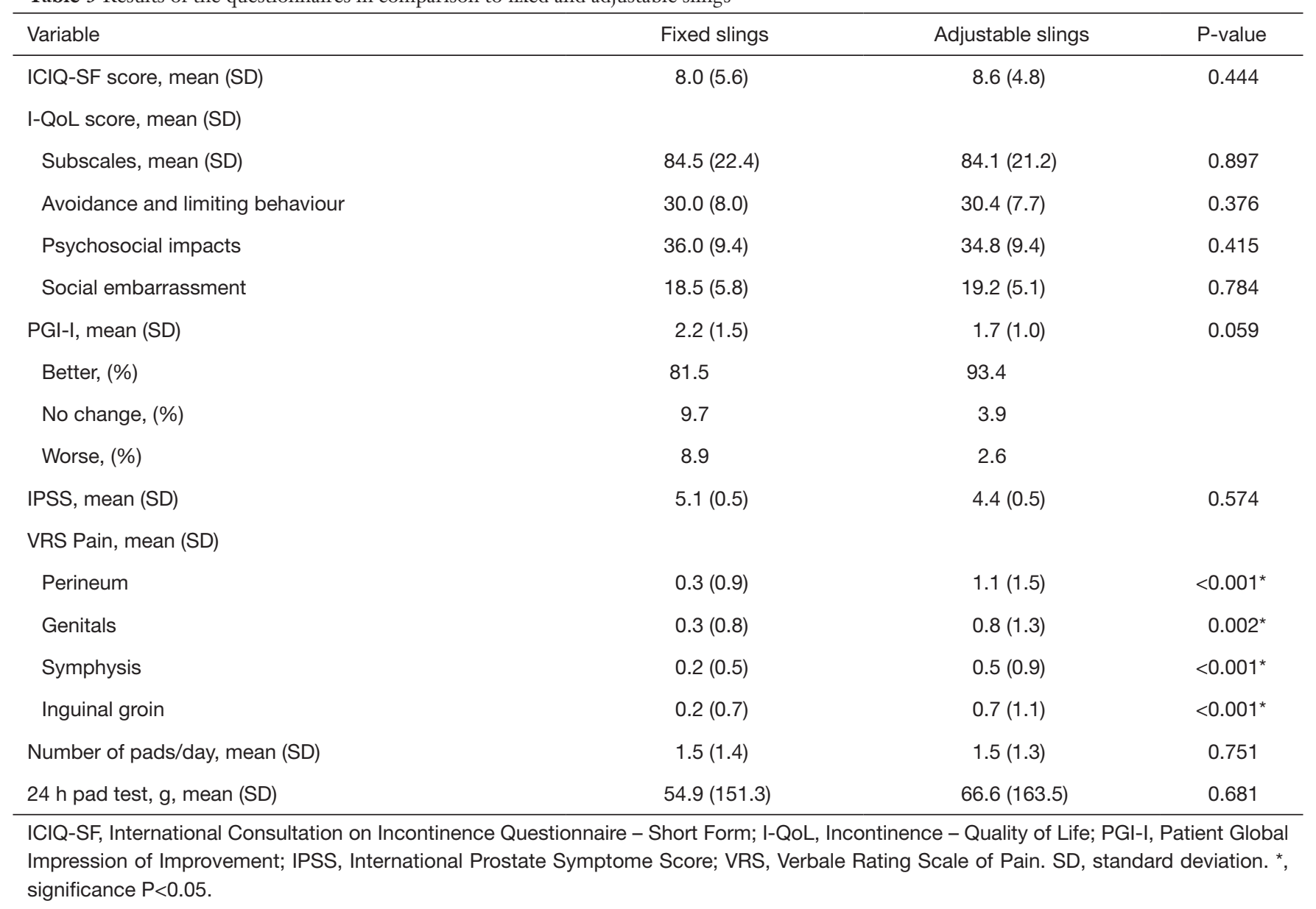

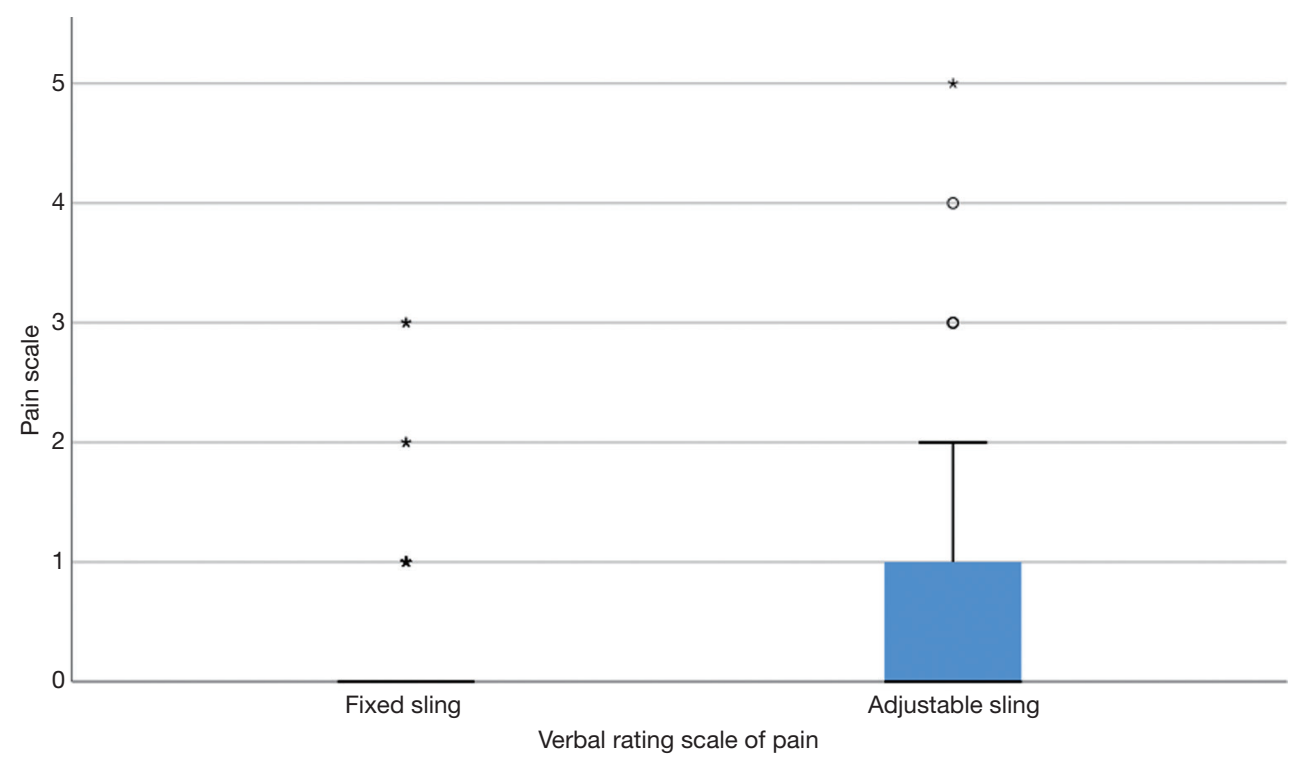

Figure 2 Perineal pain according the verbal rating scale of pain. 
included. The discrepancy according Stamey classification was marginal (1.9 vs. 2.3). The $24 \mathrm{~h}$ pad test did not demonstrate significant preoperative differences but there was a tendency for higher urine loss and larger range of urinary incontinence degree in adjustable slings. This could be interpreted in favor for adjustable slings since outcome was comparable although more risk factors were present.

The mean operation time for the adjustable MS was significantly longer, however, the mean difference was merely five minutes. The importance of this statistical difference may therefore be negligible in routine practice. Interestingly, intraoperative complications occurred in particular with the Argus classic due to bladder perforation during implantation. This might be referred to the learning curve of the surgeon or due to a particular higher risk in the retropubic route. This complication has been described before $(15,16)$ and maybe explained by perivesical scarring or even change of bladder position after radical prostatectomy. Nevertheless, the perforations were all identified and revised intraoperatively and besides prolonged postoperative catheterisation, no further treatment was necessary.

In postoperative complications, pain and infection rates were significantly higher in patients with adjustable male slings. The infections were localized at the adjustment site or at the sling arm itself. Nevertheless, infections are generally rare and the total amount of infections was $2.3 \%$ which is in line with other studies (2). Furthermore, the results may be underpowered due to the low patient number affected by infection. Regarding postoperative pain, Argus-T was particularly more frequently associated with pain which might be referred to the transobturator route and the rigid material. Nevertheless, in long term follow up this difference was not present anymore.

Pain at follow-up was significantly higher for adjustable MS. However, taking into account all anatomical sites (perineum, inguinal groin, genitals, symphysis) the mean difference amongst fixed and adjustable MS was reported between 0.8 and 0.3 . This raises the question whether or not this difference arises a clinical impact for decision making in the choice for the sling type. However, in two patients the Argus had to be even removed due to persistent pain and unchanged SUI, hereby presenting a high impact for individual cases.

In the prospective analysis of the present study, no significant differences could be identified regarding quality of life, $24 \mathrm{~h}$ pad test, pad use or satisfaction rate between fixed and adjustable slings. Considering, that patients who received an adjustable sling presented significantly more often risk factors for failure, this may imply an advantage for adjustability. Furthermore, adjustability may be an advantage in the long term in case of distension of the sling arms. This is consistent with a different trial evaluating the satisfaction and incontinence rates in comparison of Argus und AdVance in a smaller cohort with 44 patients (8). A randomized prospective trial evaluating the outcome of AdVance vs. Argus identified significant differences in the 24 h-pad test although satisfaction rates and quality of life were comparable (7). Nevertheless, only 22 patients were included and therefore the results most probably are underpowered.

We acknowledge current limitations in our study with a partially retrospective design and a mid-term follow up. Even though the multi-institutional character of the present study reflects routine practice, it involves inhomogeneous patient cohorts and operation techniques. Furthermore, selection criteria for fixed and adjustable sling was not standardized. Nevertheless, all involved institutions are experienced in the treatment of urinary male incontinence and therefore represent the current standard of care. Besides, in our opinion, the strengths of the current study are the multicenter character which reflects the actual results of clinical daily practice in Germany and Austria. The results are therefore of importance for the interpretation in routine practice.

In conclusion, patients with risk factors and a higher degree of urinary incontinence are more likely to be offered an adjustable MS. In comparison between adjustable and fixed MS, no significant differences in functional outcome and quality of life could be identified. Considering the wider indication for adjustable slings, adjustability may imply an advantage over fixed sling. However, infection as well as pain rate were significantly higher in adjustable slings implying a significant impact for the patient. Hence, adjustable male slings may be in favor in patients with risk factors and higher degree of urinary incontinence at the cost for higher risk for pain and infection. However, there is still uncertainty regarding the significance of adjustable MS in patients with mild urinary incontinence.

\section{Acknowledgments}

Parts of this study have been presented at the Annual Meeting of the European Association of Urology (EAU) in 
2019, Barcelona, Spain; Annual Meeting of the American Urology Association (AUA) in 2019, Chicago, USA and the Annual Meeting of the German Association of Urology (DGU) in 2018, Dresden, Germany.

Funding: The authors received no financial support for the research, authorship, and/or publication of this article.

\section{Footnote}

Reporting Checklist: The authors have completed the STROBE reporting checklist. Available at http://dx.doi. org/10.21037/tau-19-852

Data Sharing Statement: Available at http://dx.doi. org/10.21037/tau-19-852

Conflict of Interest: All authors have completed the ICMJE uniform disclosure form (available at http://dx.doi. org/10.21037/tau-19-852). TH reports personal fees from Promedon, outside the submitted work. TP reports personal fees from Teleflex, personal fees from Zephyr, personal fees from AMS, outside the submitted work. RA reports personal fees and other from Boston Scientific, personal fees and other from Promedon, outside the submitted work. CMN reports personal fees and other from Coloplast, outside the submitted work. WH reports nonfinancial support and other from Uromedia, non-financial support and other from Promedon, outside the submitted work. RMB reports personal fees and other from Boston Scientific, personal fees and other from Promedon, outside the submitted work. The other authors have no conflicts of interest to declare.

Ethical Statement: The authors are accountable for all aspects of the work in ensuring that questions related to the accuracy or integrity of any part of the work are appropriately investigated and resolved.

Open Access Statement: This is an Open Access article distributed in accordance with the Creative Commons Attribution-NonCommercial-NoDerivs 4.0 International License (CC BY-NC-ND 4.0), which permits the noncommercial replication and distribution of the article with the strict proviso that no changes or edits are made and the original work is properly cited (including links to both the formal publication through the relevant DOI and the license). See: https://creativecommons.org/licenses/by-nc-nd/4.0/.

\section{References}

1. Trost L, Elliott DS. Male stress urinary incontinence: a review of surgical treatment options and outcomes. Adv Urol 2012;2012:287489.

2. Hüsch T, Kretschmer A, Thomsen F, et al. Risk Factors for Failure of Male Slings and Artificial Urinary Sphincters: Results from a Large Middle European Cohort Study. Urol Int 2017;99:14-21.

3. Crivellaro S, Morlacco A, Bodo G, et al. Systematic review of surgical treatment of post radical prostatectomy stress urinary incontinence. Neurourol Urodyn 2016;35:875-81.

4. Bauer RM, Gozzi C, Klehr B, et al. AdVanceXP male sling: 2-year results of a multicentre study. World J Urol 2016;34:1025-30.

5. EAU Guidelines Urinary Incontinence 2016 [Internet]. 2016 [cited 26. April 2016]. Available online: http:// uroweb.org/guideline/urinary-incontinence/

6. Bauer RM, Soljanik I, Fullhase C, et al. Results of the AdVance transobturator male sling after radical prostatectomy and adjuvant radiotherapy. Urology 2011;77:474-9.

7. Lima JP, Pompeo AC, Bezerra CA. Argus T(R) versus Advance(R) Sling for postprostatectomy urinary incontinence: A randomized clinical trial. Int Braz J Urol 2016;42:531-9.

8. Chung E, Smith P, Malone G, et al. Adjustable versus non-adjustable male sling for post-prostatectomy urinary incontinence: A prospective clinical trial comparing patient choice, clinical outcomes and satisfaction rate with a minimum follow up of 24 months. Neurourol Urodyn 2016;35:482-6.

9. Wagner TH, Patrick DL, Bavendam TG, et al. Quality of life of persons with urinary incontinence: development of a new measure. Urology 1996;47:67-71; discussion 72.

10. Yalcin I, Bump RC. Validation of two global impression questionnaires for incontinence. Am J Obstet Gynecol 2003;189:98-101.

11. Seckiner I, Yesilli C, Mungan NA, et al. Correlations between the ICIQ-SF score and urodynamic findings. Neurourol Urodyn 2007;26:492-4.

12. Twiss CO, Fischer MC, Nitti VW. Comparison between reduction in 24-hour pad weight, International Consultation on Incontinence-Short Form (ICIQ-SF) score, International Prostate Symptom Score (IPSS), and Post-Operative Patient Global Impression of Improvement (PGI-I) score in patient evaluation after male perineal sling. Neurourol Urodyn 2007;26:8-13. 
13. Dindo D, Demartines N, Clavien P-A. Classification of Surgical Complications. Ann Surg 2004;240:205-13.

14. Hoda MR, Primus G, Fischereder K, et al. Early results of a European multicentre experience with a new self-anchoring adjustable transobturator system for treatment of stress urinary incontinence in men. BJU Int 2013;111:296-303.

15. Bauer RM, Rutkowski M, Kretschmer A, et al. Efficacy

Cite this article as: Hüsch T, Kretschmer A, Obaje A, Kirschner-Hermanns R, Anding R, Pottek T, Rose A, Olianas R, Friedl A, Homberg R, Pfitzenmaier J, Abdunnur R, Queissert F, Naumann CM, Schweiger J, Wotzka C, Nyarangi-Dix J, Hofmann T, Ulm K, Hübner W, Bauer RM, Haferkamp A; Debates On Male Incontinence (DOMINO)-Project. Fixed or adjustable sling in the treatment of male stress urinary incontinence: results from a large cohort study. Transl Androl Urol 2020;9(3):1099-1107. doi:10.21037/tau-19-852 and complications of the adjustable sling system Argus'T for male incontinence: results of a prospective 2-center study. Urology 2015;85:316-20.

16. Hübner WA, Gallistl H, Rutkowski M, et al. Adjustable bulbourethral male sling: experience after 101 cases of moderate-to-severe male stress urinary incontinence. BJU Int 2011;107:777-82. 